\title{
Metal-on-metal bonding and rebonding revisited
}

\author{
Alexander Bogicevic \\ Surface and Interface Sciences Department, Sandia National Laboratories, Albuquerque, NM 87185-1413, USA
}

(February 16, 1999)

Density-functional calculations for a wide variety of metals show that, contrary to the rebonding view of adsorbate bonding, addimers do not have notably longer surface bonds than adatoms, do not reside farther above the surface, and do not meet the rebonding arguments for augmented mobility. Rebonding concepts are found to have some utility in explaining addimer stability.

PACS number(s): $68.35 . \mathrm{Fx}, 68.35 . \mathrm{Bs}, 68.35 . \mathrm{Jk}$

\section{RECEIV MAR O 3 M9}

The bond-order-bond-length concept put forth by Pauling almost 70 years ago has greatly added to our intuitive understanding of atomic-scale bonding in molecules [1]. The basic idea is that the more bonds an atom makes, the weaker and longer each becomes. This principle is routinely used in molecular chemistry, and has also been applied to the gas-surface interface [2]. Going one step further, Feibelman has adopted similar arguments to interpret the stability and mobility of adsorbates in terms of "rebonding" [3]. At the heart of this model is the idea that an attractive interaction between adsorbates inevitably weakens their bonds to the surface. This leads to a number of important predictions [3]: (i) Addimers are expected to bind farther above the surface than adatoms because they are more coordinated. (ii) Small addimer binding energies (relative to per-bond bulk cohesive energies) result from "compensation between the cost of breaking an interadatom bond and the gain attendant on the simultaneous strengthening of adatom-surface bonds" [3]. (iii) Addimers can be more mobile than adatoms because they reside in the less corrugated potential that exists higher above the substrate, and the atom not surmounting the barrier strengthens its bond to the surface as its partner moves away [3].

Although the rebonding model provides an appealing picture of adsorbate bonding, recent first-principles adsorption calculations for $\mathrm{Al} / \mathrm{Al}(111)$ [4] disagree qualitatively with the rebonding view [5]. Since rebonding addresses fundamental issues in epitaxial growth and catalysis $[4,6]$, it is of general interest to investigate this issue $[3,4]$ in detail.

In this Letter, I show that the rebonding view of adsorbate bonding is oversimplified, and in many cases inappropriate. The notion of bond lengthening, increased resident height, and augmented mobility upon pair formation is not borne out by detailed calculations for a broad range of metal systems. The principal reason for this is that the main part of the rebonding energy lies in adsorbate and substrate relaxations that do not lengthen the adspecies-substrate bonds notably.

The calculations are based on density-functional theory (DFT) $[7,8]$, using a pseudopotential method, as implemented in the VASP code [9], and applying both the local-density (LDA) [10] and generalized gradient approximation (GGA) [11] for the exchange-correlation functional. Note that, unlike in the perturbative 1263 "post-LDA/GGA" approach, these calculations are all fully self-consistent. Both approximations to DFT are employed for the purpose of generality, and to facilitate comparisons. The one-electron wave functions are expanded in a plane-wave basis with an energy cutoff of 9 , $13,15,15 \mathrm{Ry}$ for $\mathrm{Al}, \mathrm{Au}, \mathrm{Ir}, \mathrm{Rh}$, respectively, using ultrasoft Vanderbilt pseudopotentials [13]. The Kohn-Sham equations are solved iteratively, and the atomic structure is optimized until the forces on all unconstrained atoms are less than $0.03 \mathrm{eV} / \AA$. The (100) super-cell is constructed of 8 layers each containing 20 atoms (the (111) cell has 6 layers with 30 atoms each). Above an additional adsorbate layer [14], there is $>10 \AA$ of vacuum. The surface Brillouin zone is sampled using a dense $(6 \times 6) k$-point mesh for good convergence [15]. This study focuses on the (100) face of fcc metals because the rebonding effect is expected to be pronounced on this open surface. The self-adsorption results are as follows:

Bond lengths.- A simple observable manifestation of rebonding is that addimers should reside farther above the surface than adatoms [3]. However, the present calculations, and inspection of two other unpublished studies on $\mathrm{Pt} / \mathrm{Pt}(111)$ [16] and $\mathrm{Cu} / \mathrm{Cu}(100)$ [17], argue otherwise: in all metal systems studied to date, dimers bind just as close to the surface as atoms within both the LDA and GGA. The actual adsorbate-surface bond lengths are equal for adatoms and addimers to within $0.03 \AA$ ( $\mathrm{Ta}$ ble I).

TABLE I. Adsorption parameters for adatom/addimer systems within LDA and GGA: $d$ denotes the adsorption height above the plane averaged over all surface atoms, $r$ the average bond length to the surface atoms, and $r_{l}$ the lateral bond length of the addimer. All values are in $\AA$.

\begin{tabular}{l|ccc|ccc}
\hline \hline & \multicolumn{3}{|c|}{ LDA } & \multicolumn{3}{|c}{ GGA } \\
& $d$ & $r$ & $r_{l}$ & $d$ & $r$ & $r_{l}$ \\
\hline $\mathrm{Al}(100)$ & $1.69 / 1.69$ & $2.67 / 2.69$ & 2.62 & $1.72 / 1.70$ & $2.72 / 2.73$ & 2.66 \\
$\mathrm{Au}(100)$ & $1.61 / 1.64$ & $2.69 / 2.71$ & 2.76 & $1.70 / 1.71$ & $2.77 / 2.80$ & 2.85 \\
$\mathrm{Rh}(100)$ & $1.63 / 1.64$ & $2.49 / 2.51$ & 2.57 & $1.68 / 1.69$ & $2.55 / 2.57$ & 2.64 \\
$\operatorname{Ir}(100)$ & $1.63 / 1.64$ & $2.51 / 2.53$ & 2.56 & $1.67 / 1.67$ & $2.55 / 2.56$ & 2.62 \\
$\mathrm{Al}(111)$ & $2.03 / 1.98$ & $2.65 / 2.65$ & 2.61 & $2.05 / 2.00$ & $2.69 / 2.68$ & 2.64 \\
$\operatorname{Pt}(111)^{1}$ & $1.96 / 1.96$ & $2.56 / 2.56$ & 2.63 & & & \\
\hline
\end{tabular}

${ }^{1}$ Ref. [16]. 


\section{DISCLAIMER}

This report was prepared as an account of work sponsored by an agency of the United States Government. Neither the United States Government nor any agency thereof, nor any of their employees, make any warranty, express or implied, or assumes any legal liability or responsibility for the accuracy, completeness, or usefulness of any information, apparatus, product, or process disclosed, or represents that its use would not infringe privately owned rights. Reference herein to any specific commercial product, process, or service by trade name, trademark, manufacturer, or otherwise does not necessarily constitute or imply its endorsement, recommendation, or favoring by the United States Government or any agency thereof. The views and opinions of authors expressed herein do not necessarily state or reflect those of the United States Government or any agency thereof. 


\section{DISCLAIMER}

Portions of this document may be illegible in electronic image products. Images are produced from the best available original document. 
Dimer mobility. - If addimers reside in a less corrugated potential than adatoms, it is not because of increased resident height. To test the other rebondingmodel argument for enhanced addimer mobility, I perform calculations for $\mathrm{Al} / \mathrm{Al}(100)$ in which one of the adatoms leaves the pair in a direction perpendicular to the dimer axis (as described in Ref. [3]). The activation barrier for this motion is $0.53 \mathrm{eV}$ within the GGA. The adatom that is left behind relaxes towards the adatom diffusing away, so that the average bond length to the surface actually increases by $\simeq 0.01-0.02 \AA$. Hence, neither of the two rebonding arguments for augmented addimer mobility are supported by the present calculations. Experimentally, addimers are most often found to be less mobile than adatoms [6,18]; the few exceptions are for heterogeneous systems [18], which implicates the role of addimer frustration (see below). It is possible, however, that rebonding is important for addimer diffusion by exchange (as observed for $\mathrm{Al}(100)$ and $\operatorname{Pt}(100)[19]$ ), and perhaps in concerted sliding (as predicted for $\mathrm{Al} / \mathrm{Al}(111)$ [4] and $\mathrm{Pt} / \mathrm{Pt}(111)$ [16]).

Dimer stability.- Rebonding does contribute to lowering the addimer binding energy $E_{d}$. In the few studies performed on dimer stability, $E_{d}=E_{\text {dimer }}+E_{\text {slab }}-$ $2 E_{\text {atom }}$ is routinely compared with the per-bond bulk cohesive energy $E_{c} / 6[3,18]$. In this study, as in others, $x_{2}=E_{d} /\left(E_{c} / 6\right)<1$ (Table II). An appealing feature of the rebonding model is that it suggests a simple explanation for this observation: positive adatomadatom interaction is gained at the expense of weakening adatom-surface bonds. Before assessing this effect, it is essential to realize that it is in fact misleading to expect that $E_{d}=E_{c} / 6$ for two distinct reasons. First of all, any bond strength comparisons should include the actual addimer bond strength $E_{a a}$ rather than the addimer binding energy $E_{d}$, see Fig. 1 . Upon addimer separation, each adatom retains its adatom-substrate bond $E_{a s}$, augmented by the rebonding energy $\Delta$, and hence $E_{a a}=E_{d}+2 \Delta$. Note that there is no such distinction in the gas and bulk phases, and that only in the limit of no rebonding does the addimer binding energy equal its bond strength. Secondly, the addimer bond is a trade-off

TABLE II. Binding energies within the LDA and GGA. The lattice constant $a$ is given in $\AA$, the atomic adsorption energy $E_{a}$ (calculated by subtracting the slab and spin-polarized atomic energies from the adatom system energy, c.f. Ref [12]), and addimer binding energy $E_{d}$ in $\mathrm{eV}$. The ratios between the latter two energies and calculated bulk cohesive energies are given in the last two columns: $x_{1}=E_{a} / E_{c}$ and $x_{2}=6 E_{d} / E_{c}$.

\begin{tabular}{l|ccccc|ccccc}
\hline \hline & \multicolumn{4}{|c|}{ LDA } & \multicolumn{4}{|c}{ GGA } \\
& $\mathrm{a}$ & $E_{a}$ & $E_{d}$ & $x_{1}$ & $x_{2}$ & $\mathrm{a}$ & $E_{a}$ & $E_{d}$ & $x_{1}$ & $x_{2}$ \\
\hline $\mathrm{Al}(100)$ & 3.98 & 3.68 & 0.30 & $84 \%$ & $43 \%$ & 4.04 & 3.28 & 0.26 & $84 \%$ & $43 \%$ \\
$\mathrm{Au}(100)$ & 4.07 & 3.83 & 0.27 & $87 \%$ & $37 \%$ & 4.18 & 2.80 & 0.18 & $88 \%$ & $34 \%$ \\
$\mathrm{Rh}(100)$ & 3.77 & 6.48 & 0.54 & $75 \%$ & $38 \%$ & 3.85 & 5.25 & 0.39 & $73 \%$ & $32 \%$ \\
$\mathrm{Ir}(100)$ & 3.82 & 8.33 & 0.75 & $81 \%$ & $44 \%$ & 3.89 & 7.12 & 0.58 & $82 \%$ & $40 \%$ \\
$\mathrm{Al}(111)$ & 3.98 & 3.10 & 0.52 & $74 \%$ & $75 \%$ & 4.04 & 2.71 & 0.45 & $74 \%$ & $74 \%$ \\
\hline \hline
\end{tabular}

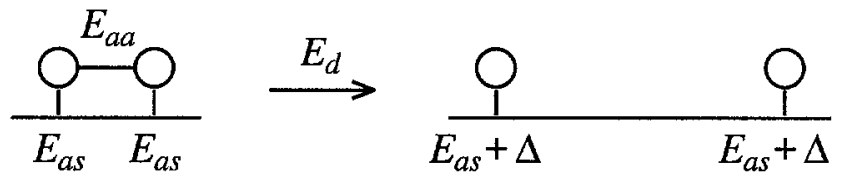

FIG. 1. The relation between the binding energy $E_{d}$, bond strength $E_{a a}$, and rebonding energy $\Delta$ of addimers.

between the covalent bond of the gas dimer and the metallic bond in the bulk (see below). Neither the binding energy nor the bond strength of the addimer should thus be expected to equal $E_{c} / 6$, just as it should not equal the gas-phase dimer bond energy.

Rebonding.- To quantitatively assess rebonding effects, I decompose the total rebonding energy into three terms, $\Delta=\Delta_{e}+\Delta_{a}+\Delta_{s}$, where the respective components are due to electronic charge redistribution, adsorbate relaxations, and substrate relaxations. These terms are calculated for $\mathrm{Al} / \mathrm{Al}(100), \mathrm{Au} / \mathrm{Au}(100)$, and $\mathrm{Rh} / \mathrm{Rh}(100)$ according to $\Delta=E_{1}-E_{21}, \Delta_{e}=E_{1}-$ $E_{11}-\left(E_{21}-E_{22}\right)$, and $\Delta_{s}=\left(2 E_{11}-E_{0}-E_{22}\right) / 2$, where the first number in the subscript denotes the number of adsorbate atoms in a fully relaxed calculation, and a second number indicates the number of adsorbate atoms removed in a calculation where the atomic coordinates have been frozen. The term $\Delta_{a}$ is obtained by subtracting $\Delta_{e}$ and $\Delta_{s}$ from $\Delta$. The results are displayed in Table III, and reveal that the rebonding energy is predominantly elastic, i.e., due to adsorbate and substrate relaxations. This explains why addimers do not bind farther away from the surface than adatoms: the (mainly lateral) relaxations do not lengthen the bonds notably. Note that the adatom strain fields are repulsive for $\mathrm{Al} / \mathrm{Al}(100)$ and $\mathrm{Rh} / \mathrm{Rh}(100)$, and attractive for $\mathrm{Au} / \mathrm{Au}(100)\left(\Delta_{s}\right.$ in Table III), which is likely to play a role in the adatom pair interaction oscillations observed on various metals [18].

Direct evidence for the lack of electronic rebonding is found in charge density analyses for $\mathrm{Al} / \mathrm{Al}(100)$, $\mathrm{Au} / \mathrm{Au}(100)$, and $\mathrm{Rh} / \mathrm{Rh}(100)$. Figure 2 shows the charge redistribution when two adatoms meet to form an addimer $\left(\rho_{2}+\rho_{22}-\rho_{21}-\rho_{21}\right)$. Note that there is practically no charge rearrangement at all in the directions towards the surface atoms (the rightmost cuts), in line with the observation that most of the rebonding is elastic. The (overall modest) charge redistribution exhibits bonding-orbital like features, and is mostly in the plane of the dimer parallel to the surface, i.e, involves orbitals which are weakly or non-bonding with respect

TABLE III. Decomposition of the rebonding energy $\Delta$, and comparison of the addimer bond strength with the calculated bulk bond strength, $x_{3}=E_{a a} /\left(E_{c} / 6\right)$ for $\mathrm{Al} / \mathrm{Al}(100)$, $\mathrm{Au} / \mathrm{Au}(100)$, and $\mathrm{Rh} / \mathrm{Rh}(100)$. All energies are in $\mathrm{eV}$.

\begin{tabular}{l|cccc|cc}
\hline \hline & $\Delta_{e}$ & $\Delta_{a}$ & $\Delta_{s}$ & $\Delta$ & $E_{a a}$ & $x_{3}$ \\
\hline $\operatorname{Al}(100)$ & $33 \%$ & $63 \%$ & $4 \%$ & 0.11 & 0.51 & $74 \%$ \\
$\operatorname{Au}(100)$ & $9 \%$ & $97 \%$ & $-6 \%$ & 0.14 & 0.57 & $77 \%$ \\
$\operatorname{Rh}(100)$ & $30 \%$ & $41 \%$ & $29 \%$ & 0.10 & 0.74 & $52 \%$ \\
\hline
\end{tabular}




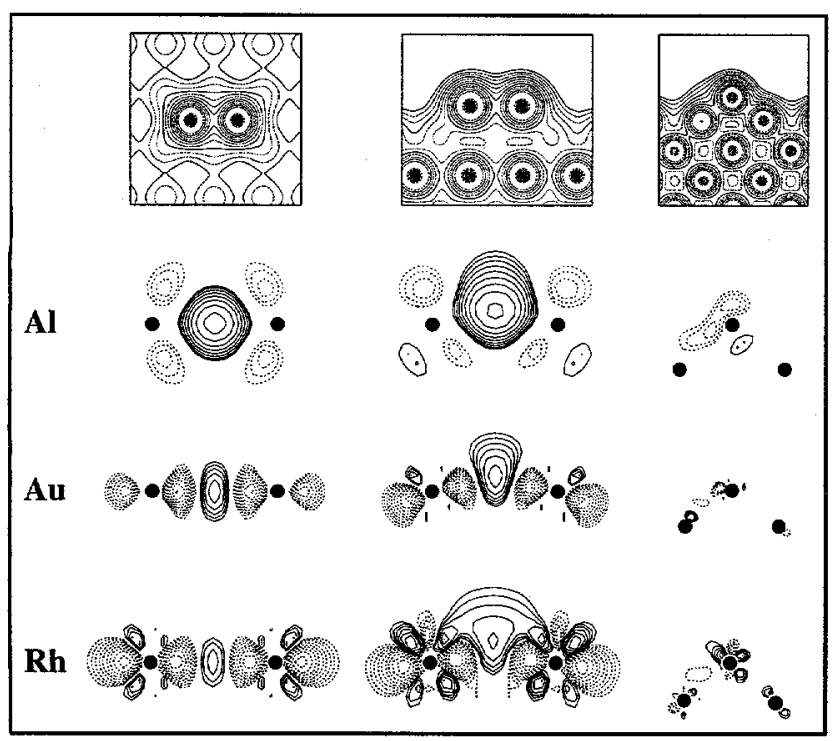

FIG. 2. Charge redistribution when two adatoms form an addimer on $\mathrm{Al} / \mathrm{Al}(100), \mathrm{Au} / \mathrm{Au}(100)$, and $\mathrm{Rh} / \mathrm{Rh}(100)$. The topmost panel shows the full charge density for $\mathrm{Au}$ to illustrate the geometry (relaxations sometimes prevent center-cuts through all atoms simultaneously). In units of $10^{-3}$ electrons $/ \AA^{3}$, the charge density range in the difference plots is $\rho=10-79(\mathrm{Al}), \rho=13-100(\mathrm{Au})$, and $\rho=20-450(\mathrm{Rh})$, and changes by a factor of $10^{0.1}\left(10^{0.15}\right)$ for $\mathrm{Al}$ and $\mathrm{Au}(\mathrm{Rh})$ between successive contours. Solid lines indicate charge accumulation, dashed lines depletion. The outermost contours are always for the lowest $\rho$, and successive contours always go towards increasing $\rho$. Atomic positions are marked by dots.

to the substrate. The reason that $\Delta_{e}$ quite generally is so small, is that the ability to form multidirectional bonds is limited both for the primarily $s-d_{\sigma}$ bonded noble and transition metals, and the $s-p$ bonded Al.

The fact that the rebonding model neglects substrate relaxations [3] is important for some metals. The relative contributions of $\Delta_{s}$ and $\Delta_{a}$ to the rebonding energy $\Delta$ vary with metal system (Table III), and indicate that neglecting substrate relaxations should be more important for the stiffer transition metals than for $\mathrm{Al}$ and $\mathrm{Au}$. For direct verification, I have performed all calculations at three levels of atomic relaxation: In the rigid case, the adsorbates are allowed to relax onto the bulk-truncated metal surface. In the frozen case, the clean slab is first relaxed, and the adsorbates then allowed to relax on this frozen-in surface. Finally, in the relaxed case (default), all atoms are allowed to relax, except for the bottom three (two for the (111) cell) layers, which are always kept at bulk positions. The results for the binding energies are displayed in Table IV, and show that substrate relaxations indeed are much more important for transition metals than for $\mathrm{Al}$ and $\mathrm{Au}$.

Returning to dimer stability, it is somewhat surprising that adatom-adatom bonds ( $E_{a a}$ in Table III) are weaker than bulk metal bonds, since they are shorter and partially covalent. Part of this oddity can be at- tributed to the higher kinetic energy associated with the more localized addimer bond, but one cannot exclude addimer "frustration" (a similar concept has been used previously in the context of cluster mobility [20]): because of their intermediate coordination, addimers strive towards a bond length somewhere between the one in the gas and bulk. Unlike in the latter two phases, however, addimer relaxation is affected by the corrugation of the surface potential. It is therefore likely that the adatom-adatom bond is frustrated in many metal systems, especially for heterogeneous adsorption. Since both compressed and dilated addimer bonds increase the system energy, the bond strength $E_{a a}$ and the binding energy $E_{d}$ of a frustrated addimer is always smaller than for the ideal tension-free addimer. Calculations for mainly heterogeneous systems are currently in progress to resolve this interesting issue.

Beyond dimers. - As deposited adatoms aggregate to form clusters, these rise up from the surface. For the systems considered here, the adsorption height of a full monolayer is $10-25 \%$ larger than for a single adatom. This difference is partly due to surface relaxations; the actual adspecies-surface bond lengths only increase by $5-6 \%$ during a monolayer formation. From simple coordination considerations, one would expect the most pronounced changes in bond length at low coordination, i.e., for addimer formation. To test this idea, I perform DFTGGA calculations for compact Al clusters on $\mathrm{Al}(100)$ containing $n=1,2,3,4,6,12$, and 20 (a full monolayer) atoms. Figure 3 shows the average adsorption height above the surface plane $d$, the average adatom-substrate bond length $r$, and the average adatom-adatom bond length $r_{l}$ in the clusters, normalized to bulk values. The most notable results are that bond lengthening proceeds rather smoothly, and that the largest changes indeed do take place for small clusters, in line with the coordination view and related effective-medium theory [21]. Since the effect of rebonding on bond lengthening is negligible where it is most pronounced, in addimer formation, it is of even less relevance to the formation of trimers, tetramers, etc. Only through the integrated effect of a large number of individual aggregations is it even observed. For the elementary atomic processes that take place in nucleation and cluster formation, the rebonding model is thus of limited value.

At this point, one might wonder how these shortcom-

TABLE IV. Dimer binding energies in eV within LDA and GGA at three levels of relaxation (see main text) indicated by the superscript: rigid $(r)$, frozen $(f)$, and fully relaxed.

\begin{tabular}{l|ccc|ccc}
\hline \hline & \multicolumn{3}{|c|}{ LDA } & \multicolumn{3}{c}{ GGA } \\
& $E_{d}$ & $E_{d}^{f}$ & $E_{d}^{r}$ & $E_{d}$ & $E_{d}^{f}$ & $E_{d}^{r}$ \\
\hline $\mathrm{Al}(100)$ & 0.30 & 0.28 & 0.26 & 0.26 & 0.22 & 0.21 \\
$\mathrm{Au}(100)$ & 0.27 & 0.28 & 0.27 & 0.18 & 0.17 & 0.18 \\
$\mathrm{Rh}(100)$ & 0.54 & 0.49 & 0.45 & 0.39 & 0.35 & 0.32 \\
$\operatorname{Ir}(100)$ & 0.75 & 0.62 & 0.60 & 0.58 & 0.46 & 0.38 \\
$\mathrm{Al}(111)$ & 0.52 & 0.48 & 0.48 & 0.45 & 0.41 & 0.41 \\
\hline \hline
\end{tabular}




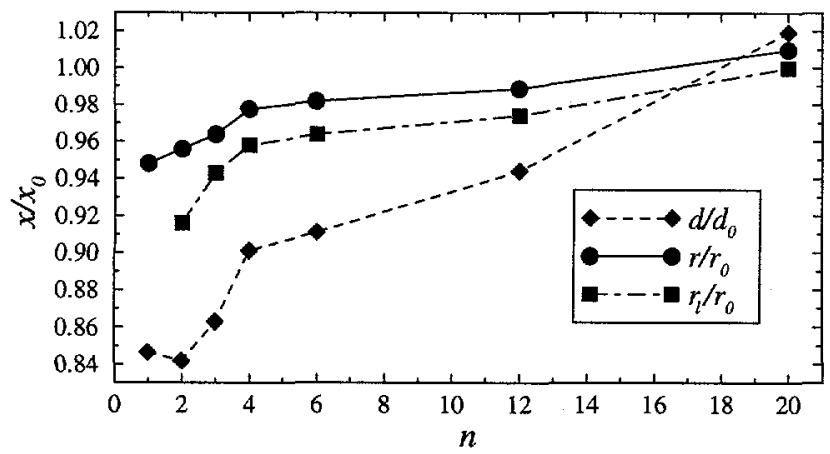

FIG. 3. Illustration of how Al clusters expand and lift off from the $\mathrm{Al}(100)$ surface as they grow. The notation is explained in the text; all bond lengths are normalized to bulk values ( $x$ on the $y$-axis stands for $d, r$, and $r_{l}$ )

ings of the rebonding model have survived previous scrutiny. The original DFT-LDA study for $\mathrm{Al} / \mathrm{Al}(100)$ persuasively argues the rebonding view, which has played an important role in establishing credibility. However, this turns out to be mainly due to a rather restricted description of the Al system: The decade-old results are based on adsorption calculations using a thin two-layer rigid substrate (due to the limited computer power at the time), that also is strained (the experimental room-temperature lattice constant is used, resulting in a tensile strain of $1.9 \%$ for the otherwise LDAdescribed system) [3]. In the previous/present study, the addimer-adatom height difference is substantial/nonexistent, $\delta d=0.16 / 0.00 \AA$, and the addimer binding energy minute/"normal", $E_{d}=0.07 / 0.30 \mathrm{eV}$ within the LDA. Using a two-layer rigid substrate without (with) $1.9 \%$ tensile strain, I find $\delta d=0.16(0.18) \AA$, and $E_{d}=0.34(0.23) \mathrm{eV}$. It is thus mainly the thin rigid slab that causes the misleadingly large $\delta d$.

In summary, I show that the rebonding view of adsorbate bonding is invalid for a wide range of metal systems. Compared with adatoms, addimers do not have notably longer surface bonds, and do not bind farther above the surface. Regarding diffusion, addimers do not experience a less corrugated potential due to increased resident height, and the atom left behind does not strengthen its surface bond as its partner moves away. Rebonding does, however, play a role for the stability of addimers, but also here there is a widespread misconception about how the bond strength should compare with bulk and gas phase interatomic bonds.

I am indebted to Dwight Jennison for illuminating discussions and good advice. I thank Per Hyldgaard, Peter Feibelman, Gary Kellogg, and Terry Michalske for valuable input, and $\mathrm{L}$. Lewis for making his $\mathrm{Pt} / \mathrm{Pt}(111)$ results available prior to publication. VASP was developed at the Institut für Theoretische Physik of the Technische Universität Wien. Sandia is a multiprogram laboratory operated by Sandia Corporation, a Lockheed Martin Company, for the United States Department of Energy under Contract DE-AC04-94AL85000. This work was partially supported by a Laboratory Directed Research and Development project.

[1] L. Pauling, J. Am. Chem. Soc. 53, 1367 (1931); The Nature of the Chemical Bond (Cornell University Press, Ithaca, NY, 1960), 3rd ed.

[2] A. R. Williams, P. J. Feibelman, and N. D. Lang, Phys. Rev. B 26, 5433 (1982); P. J. Feibelman, ibid. 35, 2626 (1987).

[3] P. J. Feibelman, Phys. Rev. Lett. 58, 2766 (1987).

[4] A. Bogicevic, P. Hyldgaard, G. Wahnström, and B. I. Lundqvist, Phys. Rev. Lett. 81, 172 (1998).

[5] The dimer binding energy for $\mathrm{Al}_{2} / \mathrm{Al}(111)$ is within $10 \%$ of the experimental per-bond bulk energy. This disagrees with rebonding theory, as pointed out by $T$. Linderoth.

[6] H. Brune, Surf. Sci. Reports 31, 121 (1998).

[7] P. Hohenberg and W. Kohn, Phys. Rev. 136, B864 (1964).

[8] W. Kohn and L. J. Sham, Phys. Rev. 140, A1133 (1965).

[9] G. Kresse and J. Hafner, Phys. Rev. B 47, 558 (1993); 49, 14251 (1994); 54, 11169 (1996).

[10] J. P. Perdew and A. Zunger, Phys. Rev. B, 23, 5048 (1981).

[11] J. P. Perdew et ol., Phys. Rev. B, 46, 6671 (1992).

[12] M. Fuchs et al., Phys. Rev. B 57, 2134 (1998).

[13] D. Vanderbilt, Phys. Rev. B 32, 8412 (1985).

[14] The artificial electric field induced by one-sided adsorption is very weak; when compensated by a dipole layer in the vacuum, energy differences change by $\lesssim 10 \mathrm{meV}$.

[15] A smaller $(4 \times 4)$ mesh yields energy differences of $\lesssim 20$ $\mathrm{meV}$ and bond length differences of $\lesssim 0.04 \AA$, and is used in the rigid and frozen (100) systems.

[16] L. J. Lewis, private communication; G. Boisvert and L. J. Lewis, to be published.

[17] P. J. Feibelman, to be published.

[18] G. Kellogg, Surf. Sci. Rep. 21, 1 (1994).

[19] G. Kellogg and P. J. Feibelman, Phys. Rev. Lett. 64, 3143 (1990); P. J. Feibelman, ibid. 65, 729 (1990); G. L. Kellogg and A. F. Voter, ibid. 67, 622 (1991).

[20] W. R. Graham and G. Ehrlich, J. Phys. F 4, L212 (1974).

[21] K. W. Jacobsen et al, Phys. Rev. B 35, 7423 (1987). 